Original article

\title{
Facile transformation of Biginelli pyrimidin-2(1H)-ones to pyrimidines. In vitro evaluation as inhibitors of Mycobacterium tuberculosis and modulators of cytostatic activity
}

\author{
Kamaljit Singh $^{\mathrm{a}, *}$, Kawaljit Singh ${ }^{\mathrm{a}}$, Baojie Wan ${ }^{\mathrm{b}}$, Scott Franzblau ${ }^{\mathrm{b}}$, Kelly Chibale ${ }^{\mathrm{c}}$, Jan Balzarini ${ }^{\mathrm{d}}$ \\ a Organic Synthesis Laboratory, Department of Applied Chemical Sciences and Technology, Guru Nanak Dev University, Amritsar 143005, India \\ ${ }^{\mathrm{b}}$ Institute for Tuberculosis Research, College of Pharmacy, University of Illinois at Chicago, 833 South Wood Street, Chicago, IL 60612-723, USA \\ ${ }^{\mathrm{c}}$ Department of Chemistry and Institute of Infectious Disease and Molecular Medicine, University of Cape Town, Rondebosch 7701, South Africa \\ ${ }^{\mathrm{d}}$ Rega Institute for Medical Research, Katholieke Universiteit Leuven, 10 Minderbroedersstraat, B-3000 Leuven, Belgium
}

\section{A R T I C L E I N F O}

\section{Article history:}

Received 18 January 2011

Received in revised form

2 March 2011

Accepted 4 March 2011

Available online 15 March 2011

\section{Keywords:}

Biginelli compounds

Pyrimidines

Tuberculosis

MABA

Inhibitors

Cytostatic activity

\begin{abstract}
A B S T R A C T
A series of pyrimidine derivatives bearing amine substituents at $\mathrm{C}-2$ position were obtained from Biginelli 3,4-dihydropyrimidin-2(1H)-ones and the effect of structural variation on anti-TB activity against Mycobacterium tuberculosis $\mathrm{H}_{37} \mathrm{Rv}$ strain and antiviral activity in a series of cell cultures was evaluated. While the compounds were found to possess structure dependent cytostatic activity, these were not found to be efficient inhibitors of $M$. tuberculosis nor did they inhibit a broad variety of DNA or RNA viruses in cell culture.
\end{abstract}

(c) 2011 Elsevier Masson SAS. All rights reserved.

\section{Introduction}

The nitrogen heterocycles in general and pyrimidines in particular are found in several biologically active natural products and depict considerable therapeutic potential [1]. In view of wide spectrum biological activities such as anti allergic [2], antitumor [3], antipyretic [4], anti-inflammatory [4] and antiparasitic [5] activities, exhibited by synthetic pyrimidine based scaffolds, a number of analogues have garnered considerable attention. During a screening effort for antiviral agents, we found that multifunctionalized tetrahydropyrimidines derivatives bearing bulky C-2 alkyl substituents depict cytostatic activity and inhibit proliferation of murine leukemia, murine mammary carcinoma, human T-lymphocyte and human cervix carcinoma cells [6]. 3,4-Dihydropyrimidin-2-(1H)-ones (DHPMs) and their appropriately functionalized derivatives have interesting pharmacological profiles [7]. These are potent antihypertensive agents, mitotic kinesin inhibitors, $\alpha_{1 \mathrm{a}}$-adrenergic receptor antagonists, or hepatitis $\mathrm{B}$ virus

\footnotetext{
* Corresponding author. Tel.: +91 183 2258853; fax: +91 183225881920 .

E-mail address: kamaljit19in@yahoo.co.in (K. Singh).
}

replication inhibitors and depict a variety of other biological effects. Although a large number of DHPM derivatives have been prepared in a single-pot Biginelli multi component reaction [8] (MCR) and its variants [9], very useful and convincing structural variability of these interesting heterocycles have been achieved through chemical functionalization of all the six positions around the DHPM core [10]. Recently, we reported [6] on the facile conversion of DHPMs into tetrahydropyrimidines. As part of an ongoing, multi-faceted program aimed toward development of small molecules as therapeutic agents, herein we report a straightforward conversion of Biginelli DHPMs into C-2 amine substituted pyrimidine derivatives and their screening as inhibitors of Mycobacterium tuberculosis and their inhibitory effect against the proliferation of some cell cultures.

\section{Chemistry}

Oxidation of DHPM derivatives 1 (Scheme 1) to 1,2-dihydropyrimidin-2-one derivatives $\mathbf{2}$ was readily achieved using pyridinium chlorochromate (PCC) as reported by us earlier [11]. Subsequent treatment of appropriate 2 with phosphorous oxychloride at $105{ }^{\circ} \mathrm{C}$ furnished corresponding 2-chloro derivatives 3a-c (Scheme 1), in 85\%, 91\% and 93\% yield, respectively. Further 


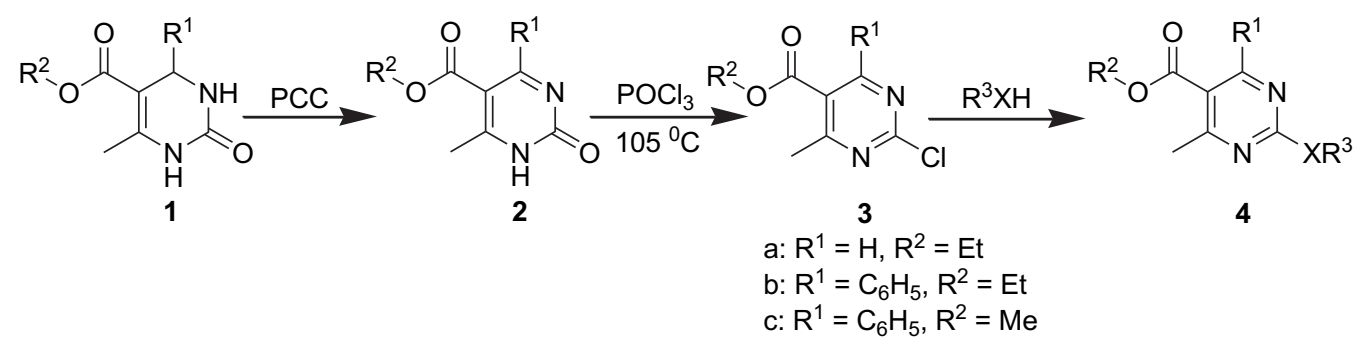

Scheme 1. Synthesis of 2-substituted pyrimidine-5-carboxylate 4 derivatives.

reaction of $\mathbf{3 a}-\mathbf{c}$ with $\mathrm{N}$ - and $\mathrm{O}$-nucleophiles, furnished corresponding 2-substituted pyrimidine derivatives $\mathbf{4}$, in very good yields (Table 1 ). This fairly simple and efficacious protocol can be visualized as a general protocol for obtaining a range of $\mathrm{C}-2$ substituted pyrimidine analogues, for obtaining C-2 diversified pyrimidine derivatives.

\section{Result and discussion}

Tuberculosis (TB) is a deadly contagious disease that spreads through the air. Tuberculosis frequently attacks lungs (as pulmonary TB) but can also affect the central nervous system, the circulatory system, genitourinary system, bones, joints and the lymphatic system. In 2008, there were estimated 8.9-9.9 million incident cases of TB, 9.6-13.3 million prevalent cases and 1.1-1.7 million deaths among HIV infected individuals. If not treated, of the more than 2 billion total individuals infected with $\mathrm{TB}$, the ones infected with active pulmonary TB are potentially contagious and pose serious threat [12]. TB is a disease of poverty affecting mostly young adults and more than half of all deaths occur in Asia. The main reasons for the rising number of people infected with TB bacterium can be attributed to wide spread intake of immunosuppressive drugs, HIV/AIDS, substance abuse, neglect or inefficient implementation of TB control programmes, poor management of TB infected patients, emergence of drug-resistant strains etc. A cumulative total of 36 million TB patients were successfully treated and over 6 million deaths were averted in Directly Observed Treatment, Short-course (DOTS) during 1995-2005 and Stop TB strategy programmes since 2006. Almost 30,000 cases of multidrug-resistant TB (MDR-TB) and extensively drug-resistant TB (XDR-TB) were notified in 2008 [12].

As the literature precedence attests [13] there is only one report on the evaluation of aniline pyrimidines [14] as antituberculosis agents but no report of the testing of 2-aminosubstituted pyrimidine 5-carboxyalte derivatives such as $\mathbf{4}$. While isoniazid (Table 2) acts through inhibition of cell-wall synthesis and inhibits InhA a NADH specific enoylase-reductase involved in the biosynthesis of fatty acids in mycobacteria [15], moxifloxacin is a broad spectrum antibacterial agent that acts through inhibition of the topoisomerase II (DNA gyrase) [16] and topoisomerase IV, required for bacterial DNA replication, transcription, repair, and recombination. PA-824 kills nonreplicating $M$. tuberculosis by intracellular NO release [17]. These examples led us to test selected derivatives 4 (Table 2) for their antituberculosis activity against $M$. tuberculosis.

From the MICs in Table 2, it can be deduced that the presence of an $\mathrm{N}$-benzyl group at $\mathrm{C}-2$ position (4d) or better a 3-aminoaniline substituent (41) seems to be useful for significant antitubercular activity. The presence of an ethyl ester, rather than methyl ester substituent at C-5 position of the pyrimidine core (4d vs $\mathbf{4 e}$ ) led to improvement in MIC values. Replacing the 3-aminoaniline substituent in $\mathbf{4 1}$ with 2-hydroxyaniline, piperidine or morpholine substituent at C-2 position to form $\mathbf{4 j}, \mathbf{4 m}$ and $\mathbf{4 n}$, respectively, only raised the MIC, without a significant effect on \% inhibition.
Replacing 3-hydroxypropyl amine substituent from C-2 position of 4f with $n$-butylamino substituent (4h) saw both an increase in \% inhibition as well as marginal decrease in MIC. However, none of these derivatives were found to be markedly active compared to the reference compounds.

While screening pyrimidine derivatives for their activity against a broad variety of DNA and RNA viruses (including HIV) in the appropriate cell culture models, we observed that while none of the C-2 alkyl/aryl/amine substituted dihydropyrimidinone derivatives showed appreciable activity against any of the investigated viruses at subtoxic concentrations, C-2 amine substituted pyrimidine derivatives $\mathbf{4 e}$ and $\mathbf{4 d}$ proved [18] markedly cytostatic against MDCK cell cultures ( IC $_{50}: 0.9 \mu \mathrm{g} / \mathrm{mL}$ and $1.2 \mu \mathrm{g} / \mathrm{mL}$, respectively). Compound 4e was more cytotoxic (MCC: $4-10 \mu \mathrm{g} / \mathrm{mL}$ ) to confluent cell cultures (i.e., human embryonic lung cells, feline Crandell kidney cells) than 4d (MCC: $\geq 100 \mathrm{mg} / \mathrm{mL}$ ) in these cell cultures. Therefore, representative members of the newly synthesized compounds $\mathbf{4}$ were evaluated for their inhibitory effect against the proliferation of murine leukemia (L1210), murine mammary carcinoma (FM3A), human T-lymphocyte (CEM), and human cervix carcinoma (HeLa) cells (Table 3 ). Whereas, pyrimidine derivatives bearing amino (4a-c, 4f-h), morpholino (4n) and ethoxy (4o) substituents at the $\mathrm{C}-2$ position (Table 1 ) depicted a marginal cytostatic activity of the test compounds ( $\mathrm{IC}_{50}: 86-500 \mu \mathrm{M}$ ), more bulky aryl group containing derivatives such as $\mathbf{4 i}-\mathbf{1}$, bearing groups such as tryptamine (4i), 2-hydroxyaniline (4j), 4-hydroxyaniline $(\mathbf{4 k})$ and 2 -aminoaniline $(\mathbf{4 1})$ rendered a significantly higher antiproliferative activity $\left(\mathrm{IC}_{50}: 13-58 \mu \mathrm{M}\right.$ ) (Table 3 ). The $\mathrm{C}-2$ piperidine substituted pyrimidine derivative $\mathbf{4 m}$ also showed higher cytostatic activity. 4-Hydroxyanilne substituted pyrimidine derivatice $4 \mathbf{k}$ showed highest cytostatic activity $\left(\mathrm{IC}_{50}: 13 \mu \mathrm{M}\right)$ against CEM cells.

Table 1

Synthesis of C-2 elaborated pyrimidine $\mathbf{4}$ derivatives.

\begin{tabular}{|c|c|c|c|c|c|c|}
\hline S. No. & Compound & $\mathrm{R}^{1}$ & $\mathrm{R}^{2}$ & $\mathrm{X}$ & $\mathrm{R}^{3}$ & Yield of $\mathbf{4}(\%)$ \\
\hline 1. & $4 a^{a}$ & $\mathrm{H}$ & $\mathrm{C}_{2} \mathrm{H}_{5}$ & $\mathrm{NH}$ & $\mathrm{H}$ & 75 \\
\hline 2. & $4 b^{a}$ & $\mathrm{C}_{6} \mathrm{H}_{5}$ & $\mathrm{C}_{2} \mathrm{H}_{5}$ & $\mathrm{NH}$ & $\mathrm{H}$ & 80 \\
\hline 3. & $4 c^{b}$ & $\mathrm{H}$ & $\mathrm{C}_{2} \mathrm{H}_{5}$ & $\mathrm{NH}$ & $\mathrm{C}_{6} \mathrm{H}_{5} \mathrm{CH}_{2}$ & 82 \\
\hline 4. & $4 d^{b}$ & $\mathrm{C}_{6} \mathrm{H}_{5}$ & $\mathrm{C}_{2} \mathrm{H}_{5}$ & $\mathrm{NH}$ & $\mathrm{C}_{6} \mathrm{H}_{5} \mathrm{CH}_{2}$ & 92 \\
\hline 5. & $4 e^{b}$ & $\mathrm{C}_{6} \mathrm{H}_{5}$ & $\mathrm{CH}_{3}$ & $\mathrm{NH}$ & $\mathrm{C}_{6} \mathrm{H}_{5} \mathrm{CH}_{2}$ & 85 \\
\hline 6. & $\mathbf{4} \mathbf{f}^{\mathrm{b}}$ & $\mathrm{C}_{6} \mathrm{H}_{5}$ & $\mathrm{C}_{2} \mathrm{H}_{5}$ & $\mathrm{NH}$ & $\left(\mathrm{CH}_{2}\right)_{3} \mathrm{OH}$ & 75 \\
\hline 7. & $4 g^{b}$ & $\mathrm{C}_{6} \mathrm{H}_{5}$ & $\mathrm{C}_{2} \mathrm{H}_{5}$ & $\mathrm{NH}$ & $\mathrm{CH}\left(\mathrm{CH}_{3}\right)_{2}$ & 87 \\
\hline 8. & $4 h^{b}$ & $\mathrm{C}_{6} \mathrm{H}_{5}$ & $\mathrm{C}_{2} \mathrm{H}_{5}$ & $\mathrm{NH}$ & $n-\mathrm{C}_{4} \mathrm{H}_{9}$ & 97 \\
\hline 9. & $4 i^{b}$ & $\mathrm{C}_{6} \mathrm{H}_{5}$ & $\mathrm{C}_{2} \mathrm{H}_{5}$ & $\mathrm{NH}$ & $\begin{array}{l}\mathrm{CH}_{2} \mathrm{CH}_{2} \\
\text { (1H-indol-3-yl) }\end{array}$ & 65 \\
\hline 10. & $4 j^{\mathrm{b}}$ & $\mathrm{C}_{6} \mathrm{H}_{5}$ & $\mathrm{C}_{2} \mathrm{H}_{5}$ & $\mathrm{NH}$ & $2-\mathrm{OHC}_{6} \mathrm{H}_{4}$ & 85 \\
\hline 11. & $4 k^{b}$ & $\mathrm{C}_{6} \mathrm{H}_{5}$ & $\mathrm{C}_{2} \mathrm{H}_{5}$ & $\mathrm{NH}$ & $4-\mathrm{OHC}_{6} \mathrm{H}_{4}$ & 94 \\
\hline 12. & $41^{\mathrm{b}}$ & $\mathrm{C}_{6} \mathrm{H}_{5}$ & $\mathrm{C}_{2} \mathrm{H}_{5}$ & $\mathrm{NH}$ & $3-\mathrm{NH}_{2} \mathrm{C}_{6} \mathrm{H}_{4}$ & 75 \\
\hline 13. & $4 m^{b}$ & $\mathrm{C}_{6} \mathrm{H}_{5}$ & $\mathrm{C}_{2} \mathrm{H}_{5}$ & $\mathrm{~N}$ & $\begin{array}{l}-\left(\mathrm{CH}_{2}\right)_{2}-\mathrm{CH}_{2}- \\
\left(\mathrm{CH}_{2}\right)_{2}-\end{array}$ & 68 \\
\hline 14. & $4 n^{b}$ & $\mathrm{C}_{6} \mathrm{H}_{5}$ & $\mathrm{C}_{2} \mathrm{H}_{5}$ & $\mathrm{~N}$ & $\begin{array}{l}-\left(\mathrm{CH}_{2}\right)_{2}-\mathrm{O}- \\
\left(\mathrm{CH}_{2}\right)_{2}-\end{array}$ & 70 \\
\hline 15. & $4 o^{\mathrm{b}}$ & $\mathrm{C}_{6} \mathrm{H}_{5}$ & $\mathrm{C}_{2} \mathrm{H}_{5}$ & 0 & $\mathrm{C}_{2} \mathrm{H}_{5}$ & 87 \\
\hline
\end{tabular}

\footnotetext{
${ }^{a} \mathrm{NH}_{3}$ gas, THF, rt
}

b $\mathrm{EtOH}, 80^{\circ} \mathrm{C}$ 
Table 2

The structure and anti-TB activity (MIC) against Mycobacterium tuberculosis for selected pyrimidine-5-carboxylate $\mathbf{4}$ derivatives.

\begin{tabular}{llc}
\hline Compound & \% Inhibition at $128 \mu \mathrm{M}$ & $\mathrm{MIC}(\mu \mathrm{M})$ \\
\hline $\mathbf{4 d}$ & 93 & 63.8 \\
$\mathbf{4 e}$ & 3 & $>128$ \\
$\mathbf{4 f}$ & 57 & $>128$ \\
$\mathbf{4 h}$ & 99 & 117.7 \\
$\mathbf{4 j}$ & 92 & 121.8 \\
$\mathbf{4 l}$ & 99 & 31.2 \\
$\mathbf{4 m}$ & 86 & $>128$ \\
$\mathbf{4 n}$ & 96 & 125.3 \\
Isoniazid & - & 0.12 \\
Moxifloxacin & - & 0.47 \\
PA-824 & - & 0.48 \\
\hline
\end{tabular}

\section{Conclusion}

In summary, a simple and efficient method for the transformation of Biginelli dihydropyrimidin-2(1H)-ones to pyrimidine derivatives bearing mainly amine substituents at $\mathrm{C}-2$ position has been devised. The methodology holds potential for the introduction of a number of tailor-made substituents at the C-2 position of DHPMs in a synthetically useful manner. While none of the compounds tested for inhibition of $M$. tuberculosis displayed useful activity, the nature of the C-2 substituent was found to modulate the cytostatic activity in cell culture.

\section{Experimental section}

\subsection{General}

All liquid reagents were dried/purified following recommended drying agents and/or distilled over 4 Å molecular sieves. THF was dried (Na-benzophenone ketyl) under nitrogen. ${ }^{1} \mathrm{H} \quad \mathrm{NMR}$ $(300 \mathrm{MHz})$ and ${ }^{13} \mathrm{C}(75 \mathrm{MHz}) \mathrm{NMR}$ spectra were recorded in $\mathrm{CDCl}_{3}$ on a multinuclear Jeol FT-AL-300 spectrometer with chemical shifts being reported in parts per million $(\delta)$ relative to internal tetramethylsilane (TMS, $\delta 0.0,{ }^{1} \mathrm{H}$ NMR) or $\left(\mathrm{CDCl}_{3}, \delta 77.0,{ }^{13} \mathrm{C} \mathrm{NMR}\right)$. Mass spectra were recorded from Indian Institute of Integrative Medicine (CSIR), Jammu, under electron impact at $70 \mathrm{eV}$ on a Bruker Daltonics Esquire 3000 spectrometer. Elemental analysis was performed on FLASH EA 112 (Thermoelectron Corporation) analyzer at Department of Chemistry, Guru Nanak Dev University, Amritsar and the results are quoted in \%. IR recorded on FTIR Shimadzu 8400

\section{Table 3}

Inhibitory effect against the proliferation of murine leukemia (L1210), murine mammary carcinoma (FM3A), human T-lymphocyte (CEM) and human cervix carcinoma (HeLa) cells.

\begin{tabular}{|c|c|c|c|c|}
\hline \multirow[t]{2}{*}{ Compound } & \multicolumn{4}{|l|}{$\mathrm{IC}_{50}^{\mathrm{a}}(\mu \mathrm{M})$} \\
\hline & L1210 & FM3A & CEM & HeLa \\
\hline $4 a$ & $245 \pm 34$ & $432 \pm 96$ & $207 \pm 45$ & $183 \pm 23$ \\
\hline $4 b$ & $402 \pm 139$ & $336 \pm 177$ & $382 \pm 166$ & $216 \pm 29$ \\
\hline $4 c$ & $378 \pm 83$ & $306 \pm 6$ & $480 \pm 29$ & $147 \pm 13$ \\
\hline $4 f$ & $238 \pm 9$ & $213 \pm 0$ & $150 \pm 33$ & $213 \pm 14$ \\
\hline $4 g$ & $132 \pm 28$ & $174 \pm 2$ & $118 \pm 44$ & $86 \pm 45$ \\
\hline $4 h$ & $164 \pm 13$ & $192 \pm 12$ & $162 \pm 40$ & $138 \pm 2$ \\
\hline $4 i$ & $28 \pm 6$ & $34 \pm 4$ & $31 \pm 3$ & $23 \pm 9$ \\
\hline $4 j$ & $58 \pm 4$ & $52 \pm 4$ & $43 \pm 3$ & $50 \pm 11$ \\
\hline $4 k$ & $24 \pm 9$ & $24 \pm 2$ & $13 \pm 4$ & $24 \pm 5$ \\
\hline 41 & $46 \pm 3$ & $43 \pm 1$ & $46 \pm 8$ & $50 \pm 2$ \\
\hline $4 m$ & $43 \pm 2$ & $55 \pm 1$ & $72 \pm 41$ & $47 \pm 3$ \\
\hline $4 n$ & $>500$ & $>500$ & $\geq 500$ & $\geq 500$ \\
\hline 40 & $251 \pm 45$ & $239 \pm 54$ & $120 \pm 66$ & $135 \pm 71$ \\
\hline
\end{tabular}

a $50 \%$ inhibitory concentration.
Fourier-transform spectrophotometer in the range $400-4000 \mathrm{~cm}^{-1}$ using chloroform as medium. Melting points were determined in open capillaries and are uncorrected. For monitoring the progress of a reaction and for comparison purpose, thin layer chromatography (TLC) was performed on pre-coated aluminium sheets Merck $\left(60 \mathrm{~F}_{254}, 0.2 \mathrm{~mm}\right)$ using an appropriate solvent system. The chromatograms were visualized under UV light. For column chromatography silica gel (60-120 mesh) was employed and eluents were ethyl acetate/hexane mixtures.

\subsection{General procedure for the synthesis of compound 3}

A solution of compound 2 (10 mmol) in phosphorous oxychloride $(10 \mathrm{~mL})$ was heated under reflux for $30 \mathrm{~min}$. The resulting reaction mixture was distilled under reduced pressure to remove excess phosphorous oxychloride. Last traces of phosphorous oxychloride were removed by azeotrophic distillation with dry benzene and crude product was purified by column chromatography to afford pure product 3.

\subsubsection{2-Chloro-4-methylpyrimidine-5-carboxylic acid ethyl ester}

(3a)

Viscous liquid. $R_{f}$ : 0.6 (25\% EtOAc/hexane). Yield: 85\%. IR ( $\mathrm{KBr}$ ): $\nu_{\max } 3350,1750,1650,650 \mathrm{~cm}^{-1} .{ }^{1} \mathrm{H}$ NMR $\left(300 \mathrm{MHz} \mathrm{CDCl}_{3}, 25^{\circ} \mathrm{C}\right)$ : $\delta 1.44\left(\mathrm{t}, J=7.2 \mathrm{~Hz}, 3 \mathrm{H}\right.$, ester- $\left.\mathrm{CH}_{3}\right), 2.84\left(\mathrm{~s}, 3 \mathrm{H}, \mathrm{C} 6-\mathrm{CH}_{3}\right), 4.42(\mathrm{q}$, $J=7.2 \mathrm{~Hz}, 2 \mathrm{H}$, ester- $\left.\mathrm{CH}_{2}\right), 9.02(\mathrm{~s}, 1 \mathrm{H}, \mathrm{C} 4-\mathrm{H}) .{ }^{13} \mathrm{C} \mathrm{NMR}(75 \mathrm{MHz}$, $\left.\mathrm{CDCl}_{3}, 25^{\circ} \mathrm{C}\right): \delta 14.0,24.6,63.6,78.5,162.0,162.8,164.7,172.5$. Anal. Calcd. for $\mathrm{C}_{8} \mathrm{H}_{9} \mathrm{~N}_{2} \mathrm{O}_{2} \mathrm{Cl}$ : C, 47.88; H, 4.49; N, 13.96; Found: C, 47.40; H, 4.10; N, 14.10. MS: $m / z 200.5\left(\mathrm{M}^{+}\right)$.

\subsubsection{2-Chloro-4-methyl-6-phenylpyrimidine-5-carboxylic acid} ethyl ester (3b)

Viscous liquid. $R_{f}$ : 0.8 (25\% EtOAc/hexane). Yield: 91\%. IR ( $\mathrm{KBr}$ ): $\nu_{\max } 3245,1740,1655,715 \mathrm{~cm}^{-1}$. ${ }^{1} \mathrm{H}$ NMR $\left(300 \mathrm{MHz}, \mathrm{CDCl}_{3}, 25^{\circ} \mathrm{C}\right)$ : $\delta 1.07\left(\mathrm{t}, J=7.2 \mathrm{~Hz}, 3 \mathrm{H}\right.$, ester- $\left.\mathrm{CH}_{3}\right), 2.61\left(\mathrm{~s}, 3 \mathrm{H}, \mathrm{C} 6-\mathrm{CH}_{3}\right), 4.20(\mathrm{q}$, $J=7.2 \mathrm{~Hz}, 2 \mathrm{H}$, ester- $\left.\mathrm{CH}_{2}\right), 7.42-7.67(\mathrm{~m}, 5 \mathrm{H}, \mathrm{ArH}) .{ }^{13} \mathrm{C} \mathrm{NMR}$ (75 MHz, $\left.\mathrm{CDCl}_{3}, 25^{\circ} \mathrm{C}\right): \delta 13.5,22.4,62.1,124.3,128.3,128.5,130.6$, 136.2, 160.4, 166.1, 166.9, 168.5. Anal. Calcd. for $\mathrm{C}_{14} \mathrm{H}_{13} \mathrm{~N}_{2} \mathrm{O}_{2} \mathrm{Cl}$ : C, 60.76; H, 4.70; N, 10.13; Found: C, 60.36; H, 4.35; N, 10.02. MS: $m / z$ $276.8\left(\mathrm{M}^{+}\right)$.

\subsubsection{2-Chloro-4-methyl-6-phenylpyrimidine-5-carboxylic acid} methyl ester (3c)

Viscous liquid. $R_{f}$ : 0.7 (25\% EtOAc/hexane). Yield: 93\%. IR ( $\mathrm{KBr}$ ): $\nu_{\max } 3245,1710,1610,665 \mathrm{~cm}^{-1}$. ${ }^{1} \mathrm{H}$ NMR $\left(300 \mathrm{MHz}, \mathrm{CDCl}_{3}, 25^{\circ} \mathrm{C}\right)$ : $\delta 2.61\left(\mathrm{~s}, 3 \mathrm{H}, \mathrm{C} 6-\mathrm{CH}_{3}\right), 3.58\left(\mathrm{~s}, 3 \mathrm{H}\right.$, ester $\left.-\mathrm{CH}_{3}\right), 7.41-7.61(\mathrm{~m}, 5 \mathrm{H}$, ArH). ${ }^{13} \mathrm{C}$ NMR ( $\left.75 \mathrm{MHz}, \mathrm{CDCl}_{3}, 25{ }^{\circ} \mathrm{C}\right): \delta 22.4,52.7,124.0,128.2$, 128.6, 130.8, 136.0, 160.5, 166.0, 167.5, 168.5. Anal. Calcd. for $\mathrm{C}_{13} \mathrm{H}_{11} \mathrm{~N}_{2} \mathrm{O}_{2} \mathrm{Cl}$ : C, 59.43; H, 4.19; N, 10.67; Found: C, 59.23; H, 4.30; N, 10.20.MS: $m / z 262.5\left(\mathrm{M}^{+}\right)$.

\subsection{Synthesis of compounds $\mathbf{4 a}$ and $\boldsymbol{b}$}

Compound 3a and $\mathbf{b}$ (1.9 mmol) was treated with THF saturated with ammonia gas (evolved by warming $30 \%$ aqueous ammonia solution) at room temperature for $45 \mathrm{~min}$. The resulting reaction mixture was distilled under reduced pressure to remove excess THF and crude product was purified through crystallization to afford compound $\mathbf{4 a}$ and $\mathbf{4 b}$.

\subsubsection{2-Amino-4-methylpyrimidine-5-carboxylic acid ethyl ester (4a)}

White solid. $R_{f}$ : 06 (60\% EtOAc/hexane). Yield: 75\%. M.p. 250-253 ${ }^{\circ} \mathrm{C}$ (Methanol). IR (KBr): $\nu_{\max } 3300,1730,1700,1450$, $1260 \mathrm{~cm}^{-1} .{ }^{1} \mathrm{H}$ NMR (300 MHz, DMSO, $\left.25^{\circ} \mathrm{C}\right): \delta 1.26(\mathrm{t}, J=6.9 \mathrm{~Hz}$, 
$3 \mathrm{H}$, ester $\left.-\mathrm{CH}_{3}\right), 2.49\left(\mathrm{~s}, 3 \mathrm{H}, \mathrm{C} 6-\mathrm{CH}_{3}\right), 4.19(\mathrm{q}, J=6.9 \mathrm{~Hz}, 2 \mathrm{H}$, ester- $\left.\mathrm{CH}_{2}\right), 7.34(\mathrm{br}, 2 \mathrm{H}, \mathrm{NH}), 8.61(\mathrm{~s}, 1 \mathrm{H}, \mathrm{C} 4-\mathrm{H}) .{ }^{13} \mathrm{C} \mathrm{NMR}(75 \mathrm{MHz}$, DMSO, $\left.25^{\circ} \mathrm{C}\right): \delta 14.1,24.1,59.9,111.4,160.8,163.7,164.8,169.6$. Anal. Calcd. for $\mathrm{C}_{8} \mathrm{H}_{11} \mathrm{~N}_{3} \mathrm{O}_{2}$ : C, 53.04; $\mathrm{H}, 6.04 ; \mathrm{N}, 23.20$; Found: C, 53.32; $\mathrm{H}$, 5.86; N, 22.90. MS: $m / z 182(\mathrm{M}+1)$.

\subsubsection{2-Amino-4-methyl-6-phenylpyrimidine-5-carboxylic acid ethyl ester (4b)}

White solid; $R_{f}$ : 0.7 (80\% EtOAc/hexane). Yield: 80\%. M.p. 290-292 ${ }^{\circ} \mathrm{C}$ (Methanol). IR (KBr): $\nu_{\max } 3409,3177,1700,1649,1550$, $1276 \mathrm{~cm}^{-1}$. ${ }^{1} \mathrm{H} \mathrm{NMR}\left(300 \mathrm{MHz}, \mathrm{CDCl}_{3}, 25^{\circ} \mathrm{C}\right): \delta 0.94(\mathrm{t}, J=7.2 \mathrm{~Hz}, 3 \mathrm{H}$, ester $\left.-\mathrm{CH}_{3}\right), 2.48\left(\mathrm{~s}, 3 \mathrm{H}, \mathrm{C} 6-\mathrm{CH}_{3}\right), 4.05\left(\mathrm{q}, J=7.2 \mathrm{~Hz}, 2 \mathrm{H}\right.$, ester $\left.-\mathrm{CH}_{2}\right)$, 5.70 (br, 2H, NH), 7.39-7.53 (m, 5H, ArH). ${ }^{13} \mathrm{C} \mathrm{NMR} \mathrm{(75} \mathrm{MHz,} \mathrm{CDCl}_{3}$, $\left.25^{\circ} \mathrm{C}\right): \delta 13.4,22.6,61.1,116.4,127.7,128.3,129.5,138.6,161.8,166.5$, 167.5, 168.4. Anal. Calcd. for $\mathrm{C}_{14} \mathrm{H}_{15} \mathrm{~N}_{3} \mathrm{O}_{2}$ : C, 65.37; $\mathrm{H}, 5.84 ; \mathrm{N}, 16.34$; Found: C, 65.20; H, 5.50; N, 15.90. MS: $m / z 258(\mathrm{M}+1)$.

\subsection{General procedure for the synthesis of compound $\mathbf{4 c - 0}$}

To a solution of compound $3(1.9 \mathrm{mmol})$ in absolute ethanol $(20 \mathrm{~mL})$ was added respective amine $(2.85 \mathrm{mmol})$ and heated at $80{ }^{\circ} \mathrm{C}$ until reaction get completed $(2-3 \mathrm{~h}$, TLC). The resulting reaction mixture was distilled under reduced pressure to remove excess ethanol and crude product was purified by column chromatography to afford pure product $\mathbf{4 c}-\mathbf{n}$ in good yield. Compound 40 was prepared by heating compound $\mathbf{3 b}(1.9 \mathrm{mmol})$ in absolute ethanol $(20 \mathrm{~mL})$ for $2 \mathrm{~h}$ at $80^{\circ} \mathrm{C}$. The resulting reaction mixture was distilled under reduced pressure to remove excess ethanol and crude product was purified by column chromatography to afford pure product $\mathbf{4 o}$.

\subsubsection{2-Benzylamino-4-methylpyrimidine-5-carboxylic acid ethyl ester (4c)}

White solid. $R_{f}: 0.8$ (20\% EtOAc/hexane). Yield: 82\%. M.p. 93-95 ${ }^{\circ} \mathrm{C}$ (DCM/Hexane). IR (KBr): $\nu_{\max } 3200,3115,1720,1545$, $1225 \mathrm{~cm}^{-1}$. ${ }^{1} \mathrm{H} \mathrm{NMR}\left(300 \mathrm{MHz}, \mathrm{CDCl}_{3}, 25^{\circ} \mathrm{C}\right): \delta 1.36(\mathrm{t}, J=7.2 \mathrm{~Hz}, 3 \mathrm{H}$, ester- $\left.\mathrm{CH}_{3}\right), 2.66\left(\mathrm{~s}, 3 \mathrm{H}, \mathrm{C} 6-\mathrm{CH}_{3}\right), 4.31(\mathrm{q}, J=7.2 \mathrm{~Hz}, 2 \mathrm{H}$, ester- $\left.\mathrm{CH}_{2}\right), 4.70\left(\mathrm{~d}, J=6.0 \mathrm{~Hz}, 2 \mathrm{H}, \mathrm{CH}_{2} \mathrm{NH}\right), 5.94(\mathrm{br}, 1 \mathrm{H}, \mathrm{NH})$, 7.24-7.36 (m, 5H, ArH), $8.85(\mathrm{~s}, 1 \mathrm{H}, \mathrm{C} 4-\mathrm{H}) .{ }^{13} \mathrm{C}$ NMR $(75 \mathrm{MHz}$, $\left.\mathrm{CDCl}_{3}, 25^{\circ} \mathrm{C}\right): \delta 14.3,24.7,29.6,45.3,60.4,127.4,127.5,128.6,138.4$, 161.1, 162.2, 165.4. Anal. Calcd. for $\mathrm{C}_{15} \mathrm{H}_{17} \mathrm{~N}_{3} \mathrm{O}_{2}$ : C, 66.42; $\mathrm{H}, 6.27 ; \mathrm{N}$, 15.50; Found: C, 66.90; H, 6.01; N, 15.18. MS: $m / z 272(\mathrm{M}+1)$.

\subsubsection{2-Benzylamino-4-methyl-6-phenylpyrimidine-5-carboxylic} acid ethyl ester $(\mathbf{4 d})$

White solid. $R_{f}$ : 0.7 (20\% EtOAc/hexane). Yield: 92\%. M.p. 70-72 ${ }^{\circ} \mathrm{C}$ (DCM/Hexane). IR (KBr): $\nu_{\max } 3258,3126,1715,1558$, $1255 \mathrm{~cm}^{-1} .{ }^{1} \mathrm{H}$ NMR $\left(300 \mathrm{MHz}, \mathrm{CDCl}_{3}, 25{ }^{\circ} \mathrm{C}\right): \delta 0.95(\mathrm{t}, J=7.2 \mathrm{~Hz}$, $3 \mathrm{H}$, ester- $\left.\mathrm{CH}_{3}\right), 2.49\left(\mathrm{~s}, 3 \mathrm{H}, \mathrm{C} 6-\mathrm{CH}_{3}\right), 4.05(\mathrm{q}, J=7.2 \mathrm{~Hz}, 2 \mathrm{H}$, ester $\left.-\mathrm{CH}_{2}\right), 4.71\left(\mathrm{~d}, \mathrm{~J}=6.0 \mathrm{~Hz}, 2 \mathrm{H}, \mathrm{CH}_{2} \mathrm{NH}\right), 5.63(\mathrm{br}, 1 \mathrm{H}, \mathrm{NH})$, 7.25-7.55 (m, 10H, ArH). $\left.{ }^{13} \mathrm{C} \mathrm{NMR} \mathrm{(75} \mathrm{MHz} \mathrm{CDCl}_{3}, 25{ }^{\circ} \mathrm{C}\right): \delta 13.5$, 22.9, 45.3, 61.0, 115.5, 127.2, 127.5, 127.9, 128.2, 128.5, 129.4, 138.9, 139.0, 161.0, 168.8. Anal. Calcd. for $\mathrm{C}_{21} \mathrm{H}_{21} \mathrm{~N}_{3} \mathrm{O}_{2}$ : C, 72.62; $\mathrm{H}, 6.05 ; \mathrm{N}$, 12.10; Found: C, 72.30; H, 5.83; N, 11.92. MS: $m / z 348$ (M+1).

\subsubsection{2-Benzylamino-4-methyl-6-phenylpyrimidine-5-carboxylic} acid methyl ester (4e)

Light brownish solid. $R_{f}$ : 0.7 (30\% EtOAc/hexane). Yield: 85\%. M.p. 103-105 ${ }^{\circ} \mathrm{C}$ (DCM/Hexane). IR (KBr): $\nu_{\max } 3261,3127,1721,1558$, $1249 \mathrm{~cm}^{-1}$. ${ }^{1} \mathrm{H}$ NMR $\left(300 \mathrm{MHz}, \mathrm{CDCl}_{3}, 25^{\circ} \mathrm{C}\right): \delta 2.47\left(\mathrm{~s}, 3 \mathrm{H}, \mathrm{C} 6-\mathrm{CH}_{3}\right)$, $3.58\left(\mathrm{~s}, 3 \mathrm{H}\right.$, ester $\left.-\mathrm{CH}_{3}\right), 4.71\left(\mathrm{~d}, J=6.0 \mathrm{~Hz}, 2 \mathrm{H}, \mathrm{CH}_{2} \mathrm{NH}\right), 5.68(\mathrm{br}, 1 \mathrm{H}$, $\mathrm{NH}), 7.23-7.55(\mathrm{~m}, 10 \mathrm{H}, \mathrm{ArH}) .{ }^{13} \mathrm{CNMR}\left(75 \mathrm{MHz}, \mathrm{CDCl}_{3}, 25^{\circ} \mathrm{C}\right): \delta 22.9$, 45.3, 51.9, 115.2, 127.2, 127.5, 127.9, 128.2, 128.5, 129.5, 138.8, 138.9, 161.1, 169.4. Anal. Calcd. for $\mathrm{C}_{20} \mathrm{H}_{19} \mathrm{~N}_{3} \mathrm{O}_{2}$ : C, 72.07; H, 5.70; $\mathrm{N}, 12.61$; Found: C, 72.23; H, 5.90; N, 12.30. MS: $m / z 334(\mathrm{M}+1)$.
5.4.4. 2-(3-Hydroxypropylamino)-4-methyl-6-phenylpyrimidine-

5 -carboxylic acid ethyl ester (4f)

Viscous liquid. $R_{f}$ : 0.3 (30\% EtOAc/hexane). Yield: 75\%. IR ( $\mathrm{KBr}$ ): $\nu_{\max } 3431,2925,1717,1597,1261,726 \mathrm{~cm}^{-1}$. ${ }^{1} \mathrm{H}$ NMR $(300 \mathrm{MHz}$, $\left.\mathrm{CDCl}_{3}, 25^{\circ} \mathrm{C}\right): \delta 0.94\left(\mathrm{t}, J=7.2 \mathrm{~Hz}, 3 \mathrm{H}\right.$, ester- $\left.\mathrm{CH}_{3}\right), 1.71-1.78(\mathrm{~m}, 2 \mathrm{H}$, $\left.\mathrm{CH}_{2} \mathrm{NH}\right), 2.50\left(\mathrm{~s}, 3 \mathrm{H}, \mathrm{C} 6-\mathrm{CH}_{3}\right), 3.59-3.68\left(\mathrm{~m}, 4 \mathrm{H}, 2 \times \mathrm{CH}_{2}\right), 4.04(\mathrm{q}$, $J=7.2 \mathrm{~Hz}, 2 \mathrm{H}$, ester $\left.-\mathrm{CH}_{2}\right), 5.59$ (br, $\left.1 \mathrm{H}, \mathrm{NH}\right), 7.39-7.53(\mathrm{~m}, 5 \mathrm{H}, \mathrm{ArH})$. ${ }^{13} \mathrm{C}$ NMR $\left(75 \mathrm{MHz}, \mathrm{CDCl}_{3}, 25{ }^{\circ} \mathrm{C}\right): \delta 13.5,22.8,33.1,37.3,58.3,61.1$, 127.7, 128.4, 129.6, 138.7, 161.7. Anal. Calcd. for $\mathrm{C}_{17} \mathrm{H}_{21} \mathrm{~N}_{3} \mathrm{O}_{3}$ : C, 64.76; H, 6.67; N, 13.33; Found: C, 64.20; H, 6.20; N, 12.90. MS: $m / z 338$ $(\mathrm{M}+23)$.

\subsubsection{2-Isopropylamino-4-methyl-6-phenylpyrimidine-5-}

carboxylic acid ethyl ester $\mathbf{( 4 g )}$

Viscous liquid. $R_{f}$ : 0.9 (20\% EtOAc/hexane). Yield: $87 \%$. IR ( $\mathrm{KBr}$ ): $\nu_{\max } 3250,1705,1590,1256,1382 \mathrm{~cm}^{-1}$. ${ }^{1} \mathrm{H}$ NMR $\left(300 \mathrm{MHz}, \mathrm{CDCl}_{3}\right.$, $\left.25{ }^{\circ} \mathrm{C}\right): \delta 0.94\left(\mathrm{t}, J=7.2 \mathrm{~Hz}, 3 \mathrm{H}\right.$, ester $\left.-\mathrm{CH}_{3}\right), 1.25(\mathrm{~d}, J=6.6 \mathrm{~Hz}, 6 \mathrm{H}$, $\left.\left(\mathrm{CH}_{3}\right)_{2} \mathrm{CH}\right), 2.47\left(\mathrm{~s}, 3 \mathrm{H}, \mathrm{C} 6-\mathrm{CH}_{3}\right), 4.04\left(\mathrm{q}, \mathrm{J}=7.2 \mathrm{~Hz}, 2 \mathrm{H}\right.$, ester $\left.-\mathrm{CH}_{2}\right)$, 4.23-4.32 (m, $\left.1 \mathrm{H}, \mathrm{CH}\left(\mathrm{CH}_{3}\right)_{2}\right), 5.40(\mathrm{br}, 1 \mathrm{H}, \mathrm{NH}), 7.38-7.53(\mathrm{~m}, 5 \mathrm{H}$, ArH). ${ }^{13} \mathrm{C}$ NMR $\left(75 \mathrm{MHz}, \mathrm{CDCl}_{3}, 25^{\circ} \mathrm{C}\right): \delta$ 13.4, 22.7, 22.9, 42.7, 60.9, 114.8, 127.8, 128.1, 129.3, 139.2, 160.4, 166.1, 167.2, 168.8. Anal. Calcd. for $\mathrm{C}_{17} \mathrm{H}_{21} \mathrm{~N}_{3} \mathrm{O}_{2}$ : C, 68.23; $\mathrm{H}, 7.02 ; \mathrm{N}, 14.05$; Found: $\mathrm{C}, 68.10 ; \mathrm{H}, 6.85$; N, 13.70.MS: $m / z 300(\mathrm{M}+1)$.

\subsubsection{2-Butylamino-4-methyl-6-phenylpyrimidine-5-carboxylic acid ethyl ester $(\mathbf{4 h})$}

Viscous liquid. $R_{f}$ : 0.8 (20\% EtOAc/hexane). Yield: 97\%. IR ( $\mathrm{KBr}$ ): $\nu_{\max } 3310,1730,1560,1382,720 \mathrm{~cm}^{-1} .{ }^{1} \mathrm{H}$ NMR $\left(300 \mathrm{MHz}, \mathrm{CDCl}_{3}\right.$, $\left.25{ }^{\circ} \mathrm{C}\right): \delta 0.92-0.97\left(\mathrm{~m}, 6 \mathrm{H}, \mathrm{CH}_{3}\right.$ and ester $\left.-\mathrm{CH}_{3}\right), 1.37-1.47(\mathrm{~m}, 2 \mathrm{H}$, $\left.\mathrm{CH}_{2}\right), 1.54-1.64\left(\mathrm{~m}, 2 \mathrm{H}, \mathrm{CH}_{2}\right), 2.48\left(\mathrm{~s}, 3 \mathrm{H}, \mathrm{C} 6-\mathrm{CH}_{3}\right), 3.46-3.52(\mathrm{~m}$, $\left.2 \mathrm{H}, \mathrm{CH}_{2} \mathrm{NH}\right), 4.04\left(\mathrm{q}, J=7.2 \mathrm{~Hz}, 2 \mathrm{H}\right.$, ester $\left.-\mathrm{CH}_{2}\right), 5.31$ (br, $1 \mathrm{H}, \mathrm{NH}$ ), $7.38-7.53(\mathrm{~m}, 5 \mathrm{H}, \mathrm{ArH}) .{ }^{13} \mathrm{C} \mathrm{NMR}\left(75 \mathrm{MHz}, \mathrm{CDCl}_{3}, 25^{\circ} \mathrm{C}\right): \delta 13.5,13.7$, 19.9, 23.0, 31.7, 41.0, 60.9, 114.9, 127.8, 128.1, 129.3, 139.2, 161.2, 166.1, 168.9. Anal. Calcd. for $\mathrm{C}_{18} \mathrm{H}_{23} \mathrm{~N}_{3} \mathrm{O}_{2}$ : C, 69.00; $\mathrm{H}, 7.35 ; \mathrm{N}, 13.42$; Found: C, 68.82; H, 6.93; N, 13.11. MS: $m / z 336$ (M+23).

\subsubsection{2-[2-(1H-Indol-3-yl)-ethylamino]-4-methyl-6-}

phenylpyrimidine-5-carboxylic acid ethyl ester (4i)

Brownish solid. $R_{f}$ : 0.5 (30\% EtOAc/hexane). Yield: 65\%. M.p. 115-117 ${ }^{\circ} \mathrm{C}$ (DCM/Hexane). IR (KBr): $\nu_{\max } 3364,3259,1693,1556$, $1261,697 \mathrm{~cm}^{-1} .{ }^{1} \mathrm{H} \mathrm{NMR}\left(300 \mathrm{MHz}, \mathrm{CDCl}_{3}, 25^{\circ} \mathrm{C}\right): \delta 0.94(\mathrm{t}, J=7.2 \mathrm{~Hz}$, $3 \mathrm{H}$, ester $\left.-\mathrm{CH}_{3}\right), 2.48\left(\mathrm{~s}, 3 \mathrm{H}, \mathrm{C} 6-\mathrm{CH}_{3}\right), 3.08\left(\mathrm{t}, J=6.9 \mathrm{~Hz}, 2 \mathrm{H}, \mathrm{CH}_{2}\right)$, $3.84\left(\mathrm{q}, J=6.9 \mathrm{~Hz}, 2 \mathrm{H}, \mathrm{CH}_{2} \mathrm{NH}\right), 4.04\left(\mathrm{q}, J=7.2 \mathrm{~Hz}, 2 \mathrm{H}\right.$, ester- $\left.\mathrm{CH}_{2}\right)$, 5.45 (br, $1 \mathrm{H}, \mathrm{NH}), 7.03-7.68(\mathrm{~m}, 1 \mathrm{H}, \mathrm{ArH}), 8.04(\mathrm{br}, 1 \mathrm{H}, \mathrm{NH}) .{ }^{13} \mathrm{C} \mathrm{NMR}$ $\left(75 \mathrm{MHz}, \mathrm{CDCl}_{3}, 25^{\circ} \mathrm{C}\right): \delta 13.5,22.9,25.4,41.5,61.0,111.1,113.0,115.1$, 118.9, 119.3, 122.0, 127.3, 127.9, 128.2, 129.3, 136.3, 139.1, 162.1, 166.1, 168.9, 172.5. Anal. Calcd. for $\mathrm{C}_{24} \mathrm{H}_{24} \mathrm{~N}_{4} \mathrm{O}_{2}$ : C, 72.00; $\mathrm{H}, 6.00 ; \mathrm{N}, 14.00$; Found: C, 71.84; H, 5.84; N, 13.83. MS: $m / z 423(\mathrm{M}+23)$.

\subsubsection{2-(2-Hydroxyphenylamino)-4-methyl-6-phenylpyrimidine-}

5-carboxylic acid ethyl ester (4j)

Yellowish solid. $R_{f}$ : 0.5 (30\% EtOAc/hexane). Yield: 85\%. M.p. $145-147{ }^{\circ} \mathrm{C}$ (DCM/Hexane). IR (KBr): $\nu_{\max } 3361,1715,1562,1258$, $737 \mathrm{~cm}^{-1}$. ${ }^{1} \mathrm{H}$ NMR $\left(300 \mathrm{MHz}, \mathrm{CDCl}_{3}, 25^{\circ} \mathrm{C}\right): \delta 0.98(\mathrm{t}, J=7.2 \mathrm{~Hz}, 3 \mathrm{H}$, ester- $\left.\mathrm{CH}_{3}\right), 2.57\left(\mathrm{~s}, 3 \mathrm{H}, \mathrm{C} 6-\mathrm{CH}_{3}\right), 4.09(\mathrm{q}, J=7.2 \mathrm{~Hz}, 2 \mathrm{H}$, ester- $\left.\mathrm{CH}_{2}\right), 6.84-7.58(\mathrm{~m}, 9 \mathrm{H}, \mathrm{ArH}), 7.27(\mathrm{br}, 1 \mathrm{H}, \mathrm{NH}), 10.23(\mathrm{br}, 1 \mathrm{H}$, $\mathrm{OH}) .{ }^{13} \mathrm{C}$ NMR $\left(75 \mathrm{MHz}, \mathrm{CDCl}_{3}, 25^{\circ} \mathrm{C}\right): \delta 13.5,22.7,61.5,120.1,120.5$, $122.2,126.0,127.2,127.8,128.5,130.2,135.0,137.6,148.6,158.6$, 167.6, 186.8. Anal. Calcd. for $\mathrm{C}_{20} \mathrm{H}_{19} \mathrm{~N}_{3} \mathrm{O}_{3}$ : C, 68.77; $\mathrm{H}, 5.44 ; \mathrm{N}, 12.03$; Found: C, 68.32; H, 5.14; N, 11.82. MS: $m / z 372$ (M+23).

\subsubsection{2-(4-Hydroxyphenylamino)-4-methyl-6-phenylpyrimidine-}

5-carboxylic acid ethyl ester (4k)

Viscous liquid. $R_{f}$ : 0.3 (30\% EtOAc/hexane). Yield: 94\%. IR ( $\mathrm{KBr}$ ): $\nu_{\max } 3365,1710,1560,1250,730 \mathrm{~cm}^{-1}$. ${ }^{1} \mathrm{H}$ NMR $\left(300 \mathrm{MHz}, \mathrm{CDCl}_{3}\right.$, 
$\left.25^{\circ} \mathrm{C}\right): \delta 0.98\left(\mathrm{t}, J=7.2 \mathrm{~Hz}, 3 \mathrm{H}\right.$, ester- $\left.\mathrm{CH}_{3}\right), 1.65(\mathrm{~s}, 1 \mathrm{H}, \mathrm{OH}), 2.54(\mathrm{~s}$, $\left.3 \mathrm{H}, \mathrm{C} 6-\mathrm{CH}_{3}\right), 4.09\left(\mathrm{q}, J=7.2 \mathrm{~Hz}, 2 \mathrm{H}\right.$, ester $\left.-\mathrm{CH}_{2}\right), 6.78-7.60(\mathrm{~m}, 9 \mathrm{H}$, ArH), 7.20 (br, $1 \mathrm{H}, \mathrm{NH}) .{ }^{13} \mathrm{C} \mathrm{NMR}\left(75 \mathrm{MHz}, \mathrm{CDCl}_{3}, 25^{\circ} \mathrm{C}\right): \delta 13.5,22.8$, 61.3, 115.5, 116.6, 121.7, 121.8, 127.9, 128.3, 129.9, 131.7, 138.6, 151.8, 158.8, 158.9, 166.0, 167.3, 168.6. Anal. Calcd. for $\mathrm{C}_{20} \mathrm{H}_{19} \mathrm{~N}_{3} \mathrm{O}_{3}$ : C, 68.77; H, 5.44; N, 12.03; Found: C, 68.90; H, 5.30; N, 11.90. MS: $m / z$ $372(\mathrm{M}+23)$.

5.4.10. 2-(3-Aminophenylamino)-4-methyl-6-phenylpyrimidine-5carboxylic acid ethyl ester (4l)

Viscous liquid. $R_{f}$ : 0.3 (20\% EtOAc/hexane). Yield: 75\%. IR ( $\left.\mathrm{KBr}\right)$ : $\nu_{\max } 3550,1705,1550,1250,735 \mathrm{~cm}^{-1}$. ${ }^{1} \mathrm{H}$ NMR $\left(300 \mathrm{MHz}, \mathrm{CDCl}_{3}\right.$, $\left.25{ }^{\circ} \mathrm{C}\right): \delta 0.98\left(\mathrm{t}, J=7.2 \mathrm{~Hz}, 3 \mathrm{H}\right.$, ester $\left.-\mathrm{CH}_{3}\right), 2.56\left(\mathrm{~s}, 3 \mathrm{H}, \mathrm{C} 6-\mathrm{CH}_{3}\right)$, $4.09\left(\mathrm{q}, J=7.2 \mathrm{~Hz}, 2 \mathrm{H}\right.$, ester- $\left.\mathrm{CH}_{2}\right), 6.38-6.41(\mathrm{~m}, 1 \mathrm{H}, \mathrm{ArH})$, 6.97-7.62 (m, 8H, ArH), 7.32 (br, $1 \mathrm{H}, \mathrm{NH}) .{ }^{13} \mathrm{C} \mathrm{NMR} \mathrm{(75} \mathrm{MHz,} \mathrm{CDCl}_{3}$, $\left.25^{\circ} \mathrm{C}\right): \delta 13.5,22.9,61.2,105.9,109.7,109.8,117.0,128.0,128.3,129.6$, 138.6, 140.1, 146.8, 158.6, 165.8, 167.1, 168.4. Anal. Calcd. for $\mathrm{C}_{20} \mathrm{H}_{20} \mathrm{~N}_{4} \mathrm{O}_{2}$ : C, 68.97; H, 5.75; N, 16.09; Found: C, 68.93; H, 5.40; $\mathrm{N}$, 15.93. MS: $m / z 349(\mathrm{M}+1)$.

5.4.11. 2-(Piperidin-1-yl)-4-methyl-6-phenylpyrimidine-5-

carboxylic acid ethyl ester (4m)

Yellowish solid. $R_{f}$ : 0.5 (10\% EtOAc/hexane). Yield: 68\%. M.p. 80-82 ${ }^{\circ} \mathrm{C}$ (DCM/Hexane). IR (KBr): $\nu_{\max } 3412,2932,2852,1718$, 1558, $700 \mathrm{~cm}^{-1}$. ${ }^{1} \mathrm{H}$ NMR (300 MHz, $\left.\mathrm{CDCl}_{3}, 25{ }^{\circ} \mathrm{C}\right): \delta 0.94(\mathrm{t}$, $J=7.2 \mathrm{~Hz}, 3 \mathrm{H}$, ester $\left.-\mathrm{CH}_{3}\right), 1.56-1.70\left(\mathrm{~m}, 3 \times 2 \mathrm{H}, \mathrm{CH}_{2}-\right.$ pipe), $2.48(\mathrm{~s}$, $\left.3 \mathrm{H}, \mathrm{C} 6-\mathrm{CH}_{3}\right), 3.88\left(\mathrm{t}, J=4.8 \mathrm{~Hz} 2 \times 2 \mathrm{H}, \mathrm{NCH}_{2}-\right.$ pipe), 4.03 (q, $J=7.2 \mathrm{~Hz}, 2 \mathrm{H}$, ester- $\left.\mathrm{CH}_{2}\right), 7.36-7.58(\mathrm{~m}, 5 \mathrm{H}, \mathrm{ArH}) .{ }^{13} \mathrm{C} \mathrm{NMR}$ ( $75 \mathrm{MHz}, \mathrm{CDCl}_{3}, 25^{\circ} \mathrm{C}$ ): $\delta 13.4,23.1,24.7,25.7,44.5,60.7,113.1,128.0$, 129.1, 139.7, 160.1, 165.6, 166.8, 169.1. Anal. Calcd. for $\mathrm{C}_{19} \mathrm{H}_{23} \mathrm{~N}_{3} \mathrm{O}_{2}$ : C, 70.15; H, 7.08; N, 12.92; Found: C, 69.91; H, 6.95; N, 12.63. MS: $m / z$ $326(\mathrm{M}+1)$.

5.4.12. 2-Morpholino-4-methyl-6-phenylpyrimidine-5-carboxylic acid ethyl ester (4n)

Yellowish solid. $R_{f}$ : 0.3 (10\% EtOAc/hexane). Yield: 70\%. M.p. 120-122 ${ }^{\circ} \mathrm{C}$ (DCM/Hexane). IR (KBr): $\nu_{\max } 2972,1718,1517$, $770 \mathrm{~cm}^{-1}$. ${ }^{1} \mathrm{H}$ NMR $\left(300 \mathrm{MHz}, \mathrm{CDCl}_{3}, 25^{\circ} \mathrm{C}\right): \delta 0.95(\mathrm{t}, J=7.2 \mathrm{~Hz}, 3 \mathrm{H}$, ester $\left.-\mathrm{CH}_{3}\right), 2.49\left(\mathrm{~s}, 3 \mathrm{H}, \mathrm{C} 6-\mathrm{CH}_{3}\right), 3.75(\mathrm{t}, J=4.5 \mathrm{~Hz} 2 \times 2 \mathrm{H}$, $\mathrm{NCH}_{2}-$ morp), 3.92 (t, $\left.J=4.5 \mathrm{~Hz}, 2 \times 2 \mathrm{H}, \mathrm{OCH}_{2}-\operatorname{morp}\right), 4.05$ (q, $J=7.2 \mathrm{~Hz}, 2 \mathrm{H}$, ester- $\left.\mathrm{CH}_{2}\right), 7.38-7.58(\mathrm{~m}, 5 \mathrm{H}, \mathrm{ArH}) .{ }^{13} \mathrm{C} \mathrm{NMR}$ (300 MHz, $\mathrm{CDCl}_{3}, 25^{\circ} \mathrm{C}$ ): $\delta 13.4,23.1,44.0,60.9,66.8,114.4,128.0$, 129.3, 139.2, 160.1, 165.6, 166.9, 168.9. Anal. Calcd. for $\mathrm{C}_{18} \mathrm{H}_{21} \mathrm{~N}_{3} \mathrm{O}_{3}$ : C, 66.06; H, 6.42; N, 12.84; Found: C, 65.72; H, 6.21; N, 12.43 . MS: $m / z 328(\mathrm{M}+1)$.

\subsubsection{2-Ethoxy-4-methyl-6-phenylpyrimidine-5-carboxylic acid} ethyl ester (4o)

Viscous liquid. $R_{f}$ : 0.3 (10\% EtOAc/hexane). Yield: $87 \%$. IR ( $\mathrm{KBr}$ ): $\nu_{\max } 3300,1730,1700,1150,1410 \mathrm{~cm}^{-1}$. ${ }^{1} \mathrm{H}$ NMR $\left(300 \mathrm{MHz}, \mathrm{CDCl}_{3}\right.$, $\left.25{ }^{\circ} \mathrm{C}\right): \delta 1.03\left(\mathrm{t}, J=6.9 \mathrm{~Hz}, 3 \mathrm{H}\right.$, ester- $\left.\mathrm{CH}_{3}\right), 1.45(\mathrm{t}, J=6.9 \mathrm{~Hz}, 3 \mathrm{H}$, $\left.\mathrm{CH}_{3}\right), 2.57\left(\mathrm{~s}, 3 \mathrm{H}, \mathrm{C} 6-\mathrm{CH}_{3}\right), 4.15\left(\mathrm{q}, J=7.2 \mathrm{~Hz}, 2 \mathrm{H}\right.$, ester- $\left.\mathrm{CH}_{2}\right), 4.50$ $\left(\mathrm{q}, J=7.2 \mathrm{~Hz}, 2 \mathrm{H}, \mathrm{OCH}_{2}\right), 7.40-7.64(\mathrm{~m}, 5 \mathrm{H}, \mathrm{ArH}) .{ }^{13} \mathrm{C} \mathrm{NMR}(75 \mathrm{MHz}$, $\left.\mathrm{CDCl}_{3}, 25^{\circ} \mathrm{C}\right): \delta 13.4,14.3,22.6,61.4,63.5,119.6,128.1,129.9,137.7$, 163.9, 166.3, 168.2, 168.5. Anal. Calcd. for $\mathrm{C}_{16} \mathrm{H}_{18} \mathrm{~N}_{2} \mathrm{O}_{3}$ : C, 67.13; $\mathrm{H}$, 6.29; N, 9.79; Found: C, 66.82; H, 6.10; N, 9.45. MS: m/z 309 (M+23).

\subsection{Antituberculosis activity assays}

Minimum inhibitory concentrations (MIC) against replicating cultures of M. tuberculosis ATCC 27294 (American Type Culture Collection, Rockville, MD) were determined after 7 days incubation with test samples in 7H12 medium [19] using the microplate Alamar Blue assay [20] (MABA). The MIC was defined as the lowest concentration effecting a reduction of $\geq 90 \%$ in fluorescence relative to untreated controls.

\subsection{Cytostatic activity assays}

Murine leukemia L1210, murine mammary carcinoma FM3A, human T-lymphocyte CEM and human cervix carcinoma HeLa cells were suspended at 300,000-500,000 cells/mL in RPMI-1640 culture medium supplemented with $10 \%$ fetal bovine serum and $2 \mathrm{mM}$ L-glutamine, and $100 \mu \mathrm{l}$ of the cell suspensions were added to $100 \mu \mathrm{l}$ of an appropriate dilution of the test compounds in 96-wellmicrotiter plates. After incubation at $37{ }^{\circ} \mathrm{C}$ for two (L1210 and FM3A) or three (CEM and HeLa) days, the cell number was determined using a Particle Counter ZI (Coulter, Analis, Ghent, Belgium). The number of the suspension cells could be counted directly; the number of the monolayer HeLa cells was counted after detachment of the cells upon trypsinization. The $\mathrm{IC}_{50}$ was defined as the compound concentration required for inhibiting cell proliferation by $50 \%$.

\section{Acknowledgments}

The authors are grateful for the financial support from UGC (F. No. 37-188/2009(SR)), CSIR (01(2364)/10/EMR-II) and K.U. Leuven (GOA no. 10/14). KS thanks CSIR for a senior research Fellowship.

\section{References and notes}

[1] D.T. Hurst, Introduction to the Chemistry and Biochemistry of Pyrimidines, Purines and Pteridines. John Wiley \& Sons, New York, 1980.

[2] M. Ban, H. Taquchi, T. Katsushima, S. Akoki, A. Wantanbe, Bioorg. Med. Chem. 6 (1998) 1057-1067.

[3] G.E. Wright, J.J. Gombino, J. Med. Chem. 27 (1984) 181-185.

[4] L.F. Jalander, J.E. Longquist, Heterocycles 48 (1998) 743-747.

[5] S.K. Srivastva, A. Agarwal, P.K. Murthy, P.M.S. Chauhan, S.K. Agarwal, A.P. Bhaduri, S.N. Singh, N. Fatima, R.K. Chatterjee, J. Med. Chem. 42 (1999) 1667-1672.

[6] K. Singh, D. Arora, J. Balzarini, Tetrahedron 66 (2010) 8175-8180.

[7] (a) C.O. Kappe, Eur. J. Med. Chem. 35 (2000) 1043-1052;

(b) K. Singh, D. Arora, K. Singh, S. Singh, Mini Rev. Med. Chem. 9 (2009) 95-106.

[8] P. Biginelli, Gazz. Chim. Ital. 23 (1893) 360-416.

[9] C.O. Kappe, Tetrahedron 49 (1993) 6937-6963.

[10] (a) K. Singh, D. Arora, E. Poremsky, J. Lowery, R.S. Moreland, Eur. J. Med. Chem. 44 (2009) 1997-2001;

(b) K. Singh, D. Arora, D. Falkowski, Q. Liu, R.S. Moreland, Eur. J. Org. Chem. (2009) 3258;

(c) K. Singh, S. Singh, Tetrahedron 65 (2009) 4106-4112 and references cited therein.

[11] K. Singh, K. Singh, Aust. J. Chem. 61 (2008) 910-913.

[12] (a) Global Tuberculosis Report: A Short Update to the 2009 Report. WHO Geneva, Switzerland, 2009;

(b) D. Maher, M.C. Raviglionem, in: D. Schlossberg (Ed.), Tuberculosis and Nontuberculosis, Mycobacterial Infections, fourth ed. Saunders, Philadelphia, 1999, p. 104;

(c) B.R. Bloom, C.J.L. Murray, Science 257 (1992) 1055-1064.

[13] Y.L. Janin, Bioorg. Med. Chem. 15 (2007) 2479-2513.

[14] J. Morgan, R. Haritakul, P.A. Keller, Bioorg. Med. Chem. Lett. 13 (2003) $1755-1757$.

[15] K. Johnsson, D.S. King, P.G. Schultz, J. Am. Chem. Soc. 117 (1995) 5009-5010.

[16] K. Drlica, X. Zhao, Microbiol. Mol. Biol. Rev. 61 (1997) 377-392.

[17] R. Singh, U. Manjunatha, H.I.M. Boshoff, Y.H. Ha, P. Niyomrattanakit, R. Ledwidge, C.S. Dowd, I.Y. Lee, P. Kim, L. Zhang, S. Kang, T.H. Keller, J. Jiricek, C.E. Barry, Science 322 (2008) 1392-1395.

[18] K. Singh, K. Singh, J. Balzarini, Unpublished results.

[19] K. Falzari, Z. Zhu, D. Pan, H. Liu, P. Hongmanee, S.G. Franzblau, Antimicrob. Agents Chemother. 49 (2005) 1447-1454.

[20] L. Collins, S.G. Franzblau, Antimicrob. Agents Chemother. 41 (1997) 1004-1009. 